\title{
VARIATION IN LENGTH OF HEAT PERIODS IN ALBINO RATS ACCORDING TO AGE
}

\author{
E. W. RASMUSSEN AND B. R. KAADA \\ Neurophysiological Laboratory, Anatomical Institute, \\ University of Oslo, Norway
}

(Received 8th Fuly 1964, revised 30th December 1964)

\begin{abstract}
Summary. The length of the cyclic heat periods of virgin albino rats was found to increase from the age of 5 to 7 months to 15 to 16 months. There was no change in the length of the oestrous cycle. From the age of 17 months the duration of each heat period and the number of females displaying heat behaviour were again reduced. Identical physiological changes in the pattern of heat were found in multiparae. Females with a short heat period exhibit a stronger approach tendency towards a sex incentive during heat than the ones in which the heat lasts for several days.
\end{abstract}

\section{INTRODUCTION}

In a study of the influence of brain lesions on the approach behaviour in response to a sex incentive an increase in the length of the heat period was noted in some female albino rats following the operation (unpublished data). This increase was at first assumed to be caused by the brain lesions. However, since the phenomenon could not be reproduced in other females subjected to apparently identical brain lesions, and since a lengthening of the heat period occasionally occurred also among the normal control rats, a study of the relationship between heat pattern and age was undertaken. In addition, the experiments have given information on the relation between heat pattern and the strength of approach behaviour towards a sex incentive.

The various stages of the reproductive cycle of female rats were found by Long \& Evans (1922) to be correlated with characteristic changes in the vaginal mucosa, uterus and follicles. In the stage of heat (oestrus) there is, according to these investigators, a complete cornification of the vaginal mucosa. Subsequent studies of the behavioural manifestations during the different stages of the oestrous cycle have shown that the copulatory response represents a better index of heat than does cornification. Hemmingsen (1933) has given a detailed description of heat behaviour and has introduced a twelve-point rating scale to characterize the intensity of heat. According to this classification of the various degrees of female sexual behaviour, heat is not always associated with cornification of vaginal epithelium. Young, Boling \& Blandau (1941) observed that in most rats the heat period began with the appearance of the first cornified cells and was nearly over at the stage of complete cornification. The latter coincided 
with ovulation, which in most rats follows heat. Thus, complete cornification is more indicative of ovulation than of heat. In the present study the classification of Hemmingsen was utilized.

\section{Heat behaviour}

\section{METHOD}

For a detailed description of Hemmingsen's classification of heat behaviour the reader is referred to the original work (1933, p. 119-124). This classification is summarized by Munn (1950). In brief, in Stages 1 and 2 of Hemmingsen's twelve-point rating scale the female is non-receptive, runs away from the male and kicks back, usually making it impossible for the male to mount. The Stages 3 to 12 represent various degrees of sexual receptivity. Females in intense heat display a characteristic behaviour: when tickled along the posterior part of the back by the investigator's fingers, the female shows quivering of the ears, darting movements, quick leaps and sudden stops with vibration of the ears or quaking of the whole body, and frequently also lordosis. When elicited in the absence of a male, these responses represent the highest degrees of receptivity, namely Stages 10 to 12 in Hemmingsen's classification. In Stages 3 to 9 a variety of these responses is provoked only when the female is mounted by a male.

Albino rats (147 virgins and eleven multiparae) from a randomly bred strain maintained in our laboratory were used. For each period of examination the animals were tested on twelve consecutive nights, which is the time when albino rats exhibit their greatest sexual activity. All observations were made after midnight. The type of behaviour in response to tickling of the posterior part of the back was first recorded. When a female in her home cage did not react to such stroking, she was put into the cage of a potent male and the type of behaviour classified according to Hemmingsen's scale. The female was promptly removed before a successful copulation took place.

\section{Approach behaviour}

The group of forty-seven females observed for sexual receptivity at the age of 12 to 13 months was also tested for approach behaviour towards a sex incentive. An obstruction box method was used in which the number of crossings of an electrified grid towards a male in a given period (30 $\mathrm{min}$ ) was used as a measure of the approach tendency.

\section{Virgins}

\section{RESULTS}

Age 5 to 7 months. A total of one hundred females of this age was studied. Text-fig. I (A to D) shows examples of the typical oestrous cycle of females in heat regularly every 5 th (A), 4th (B and C) or 3rd (D) night. In each cycle, the rats were ordinarily in heat for one night only and exhibited sexual nonreceptivity (Stage 1 or 2) on the intervening nights. From Text-fig. 2 (solid columns) it is seen that, in the 12-night period, $38 \%$ of the females of this group showed sexual receptivity on 3 nights (a cycle of 4 nights); $25 \%$ of the females were in heat on 2 nights, and $27 \%$ on 1 night only. Six per cent of the females 


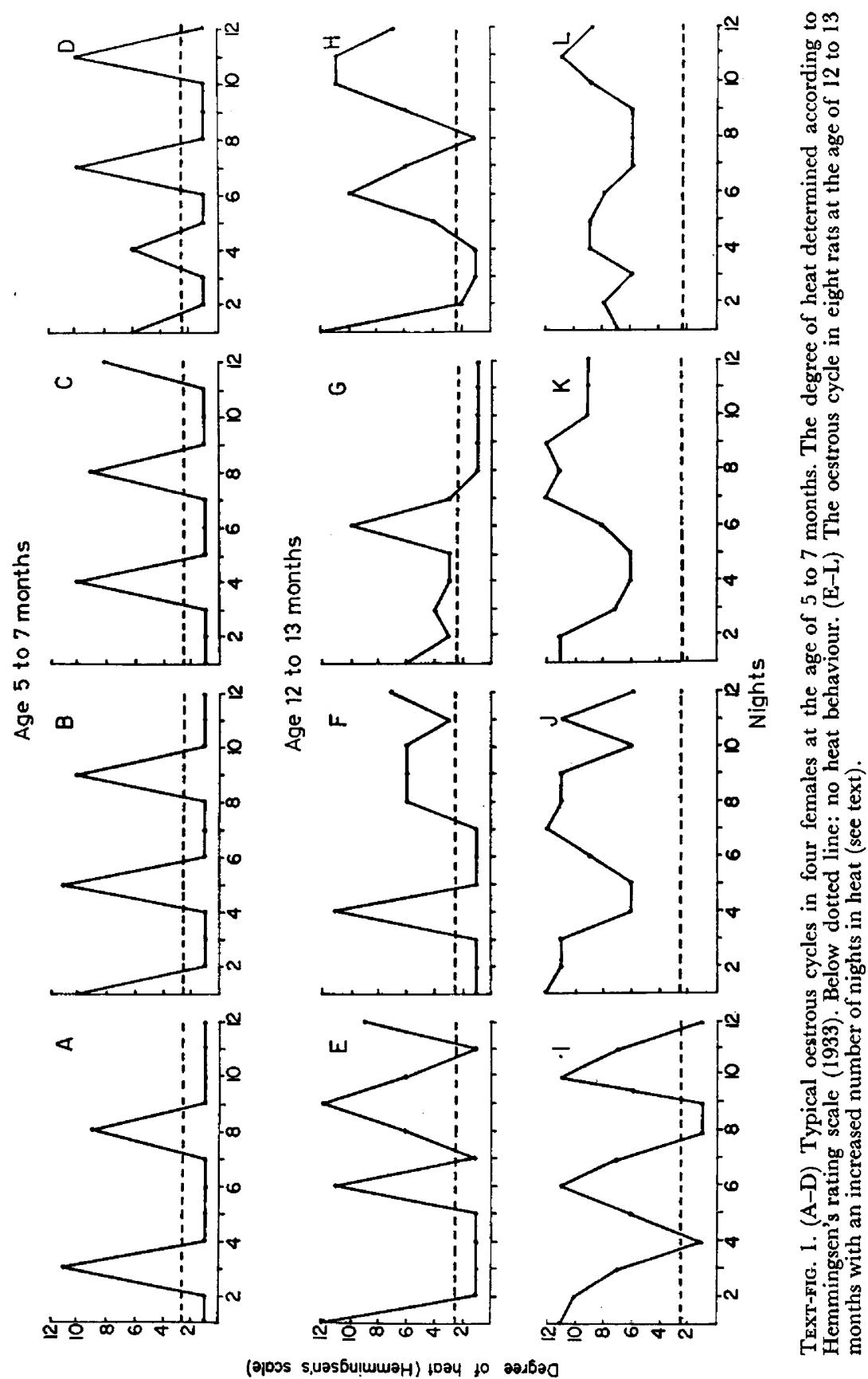


did not exhibit any heat behaviour, $3 \%$ presented the signs of heat as often as 4 nights, and $1 \%$ up to 5 nights. For the entire group the mean number of nights of heat in the 12-night period was 2.1.

Age 12 to 13 months. Observations were made in another group of forty-seven females aged 12 to 13 months. In this group, there was a significant increase in the number of nights in heat in a 12-night period. The mean was 3.4 nights, as compared to $2 \cdot 1$ for the group of half-year old rats presented above. It is seen from Text-fig. 2 that, whereas none of the younger rats were in heat for more than 5 nights in the 12-night period, nine out of forty-seven rats of this second group (19.2\%) displayed sexual receptivity more often. Six per cent of the animals were even in continuous heat. However, these animals presented higher and lesser degrees of sexual receptivity, thus indicating cyclic variations as in the first group.

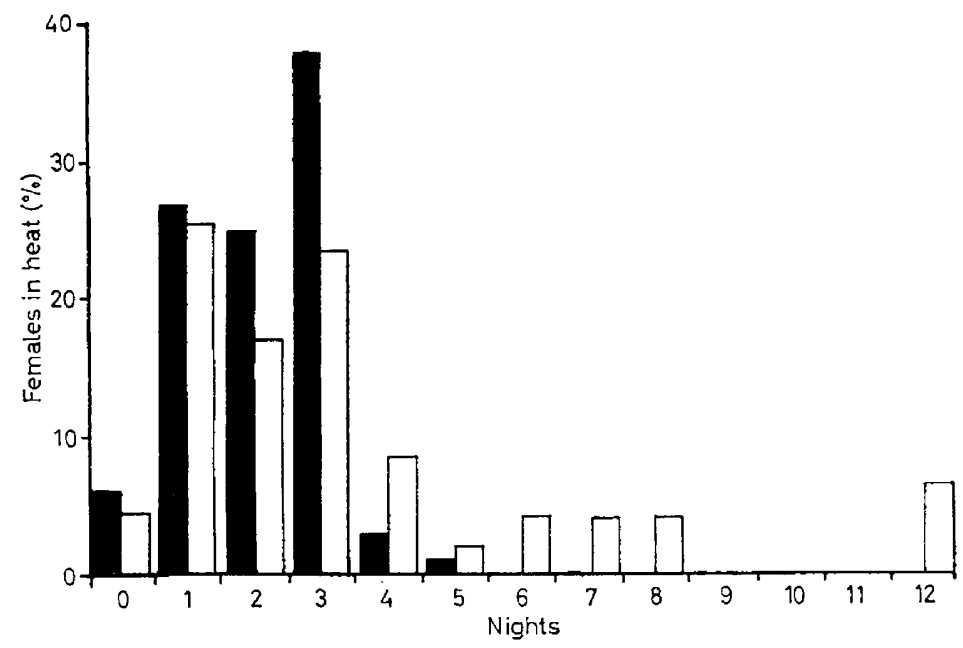

TEXT-FIG. 2. Frequency distribution of the females at the age of 5 to 7 months (100 animals) (solid columns) and 12 to 13 months (forty-seven animals) (open columns), in relation to the number of nights in heat in a 12-night period.

Text-fig. 1 ( $E$ to $L$ ) shows examples of the oestrous cycle in eight rats with lengthening of the heat period: two animals were in heat on 6 nights $(E, F)$, one on 7 nights $(G)$, one on 8 nights $(H)$, one on 9 nights $(I)$, and three were in continuous heat $(J, K, L)$. The oestrous cycle in these l-year old rats appeared to be more irregular than in the younger animals. Since the total number of peaks of sexual receptivity was approximately the same $(2 \cdot 1)$ in the 1-year old group as the half-year old animals, it is the total duration of each heat period that has increased, while there is no change in the length of the oestrous cycle.

An increase in the duration of the heat period does not necessarily mean a corresponding enhancement of the approach tendency towards a sex incentive. In the obstruction test the number of crossings was compared in two groups of rats at age of 12 to 13 months, each group belonging to opposite extremes with regard to heat duration. In twelve females found in heat on only 1 night in a 
12-night period, the mean number of crossings in the obstruction box was 12.3. In another group of nine females, exhibiting heat behaviour on 6 to 12 nights in the 12-night observation period, the mean number of crossings was $6 \cdot 0$. The obstruction test was performed on the night when the female displayed a high level of sexual receptivity, i.e. stages 10 to 12 of Hemmingsen.

These results indicate that females which are seldom in heat exhibit a stronger approach tendency towards a sex incentive, as measured by the obstruction method, compared to rats having a longer heat duration.

Age 15 months. Thirty-four of the forty-seven rats of the second group were used in other experiments, whereas the remaining thirteen were retested for sexual receptivity at the age of 15 months. No significant change in the individual rats was found in the length of the heat period. The same three rats which were in continuous heat at the age of 12 months were still in continuous heat 3 months later.

Age 17 months. Twelve out of the thirteen rats mentioned above were again tested over a 12-night period at the age of 17 months. The mean number of nights in heat now had dropped to about one-half of its former value calculated 2 months before. This also applied to the three rats previously found to be in continuous heat. In one-half of the twelve rats heat was completely absent.

To test whether this behavioural response really corresponded to oestrus, vaginal smears were secured from two of these rats at the end of the observation period on 6 nights when in heat. In eleven of these twelve smears, cornified cells dominated the picture (oestrus or pro-oestrus), whereas one rat was in a questionable anoestrus. Ordinarily rats in a definite anoestrus, as judged from the vaginal smears, are non-receptive.

Age 21 months. A fourth examination revealed for the entire group a further reduction in the length of the heat period, the mean number of nights in heat now being reduced to 1.4 in the 12-night period.

\section{Multiparae}

A group of eleven females, having bred, was also observed and tested for the first time at the age of 16 months. Ten of these rats had had litters three times, and one female, twice. The mean number of nights in heat during a 12-night period was $4 \cdot 0$. Two of these eleven multiparae were found in continuous heat; the others displayed sexual receptivity in less than 5 nights in the 12-night period. The animals were re-examined at the age of 23 months and the mean number of nights in heat had now dropped to $2 \cdot 2$.

Thus, multiparae show similar age variations as virgins with respect to the length of the heat period.

\section{DISCUSSION}

The present study demonstrates that the duration of heat period in albino rats increases with age up to 15 to 16 months, a physiological phenomenon which to our knowledge has not been described previously. Six per cent of the females even displayed a continuous heat at this age. This prolonged or persistent heat behaviour was associated with a corresponding pro-oestrus or oestrus of the vaginal cycle. This eliminates the possibility that the increased heat duration 
is produced by any other factor than a hormonal one, such as training. Anoestrus is regularly associated with sexual non-receptivity. It is of importance to bear in mind this change in the duration of oestrus according to age in studies of factors influencing the oestrous cycle in albino rats, virgins or parous. On the other hand, females displaying a prolonged heat behaviour exhibit a weaker approach tendency towards a sex incentive in the obstruction box at peak periods.

From the age of 16 to 17 months, the prolonged heat periods are followed by a shortening of such periods and a decrease in the number of females displaying heat behaviour. These changes in old rats correspond to those observed by Thung, Boot \& Mühlbock (1956) in old mice. The latter exhibited irregularities in cyclic changes of the vaginal smears consisting mainly in progressively lengthening anoestrous and metoestrous intervals with a gradual decrease of the oestrous cycle towards the end of life.

\section{ACKNOWLEDGMENTS}

This investigation was sponsored in part by the Air Force Office of Scientific Research, OAR, through the European Office, Aerospace Research, United States Air Force, under Contract AF 61 (514)-1127, and in part by the Norwegian Research Council for Science and the Humanities.

\section{REFERENGES}

Hemmingsen, A. M. (1933) Studies on the oestrus-producing hormone (oestrin). Skand. Arch. Physiol. $65,97$.

Long, J. A. \& Evans, H. M. (1922) The oestrus cycle in the rat and its related phenomena. University of California Press, Berkley.

Munn, N. L. (1950) Handbook of psychological research on the rat, p. 28. Houghton Mifflin, Boston.

Thung, P. J., Воот L. M. \& Müнцвоск, O. (1956) Senile changes in the oestrous cycle and in ovarian structure in some inbred strains of mice. Acta endocr. (Kbh.), 23, 8.

Young, W. C., Boling, J. K. \& Blandat, R. J. (1941) The vaginal smear picture, sexual receptivity and time of evulation in the albino rat. Anat. Rec. 80, 37. 